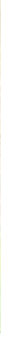

\title{
LIANAS EM UM FRAGMENTO FLORESTAL URBANO EM RIO BRANCO, ACRE, BRASIL
}

Lianas in an urban forest fragment in Rio Branco, Acre, Brazil

Lianas en un fragmento forestal urbano en Río Branco, Acre, Brasil

Lucélia Rodrigues SANTOS

Mestre em Botânica, Universidade Paulista - UNIP

santos.luceliarodrigues@gmail.com

\section{Evandro José Linhares FERREIRA}

Dr. em Botânica, Instituto Nacional de Pesquisas da Amazônia evandro@inpa.gov.br

\section{Ednéia Araújo dos SANTOS}

Mestre em Botânica, Herbário da Universidade Federal do Acre - UFAC edneiasantos_14@hotmail.com

\section{Simone Pereira da SILVA}

Especialista em Gestão Florestal, Herbário da Universidade Federal do Acre - UFAC simone.psm@gmail.com

\section{Cleison Cavalcante de MENDONÇA}

Engenheiro Florestal, Herbário da Universidade Federal do Acre - UFAC cl_cmendonca@hotmail.com 


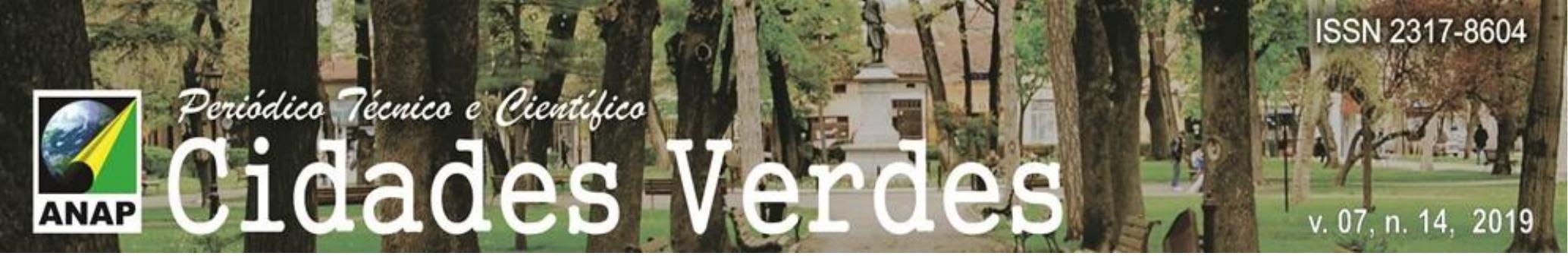

\section{RESUMO}

Este estudo objetivou avaliar os aspectos florísticos e ecológicos das lianas ocorrentes no fragmento florestal da Área de Proteção Ambiental Raimundo Irineu Serra, em Rio Branco, Acre. Realizou-se o inventário florístico com alocação de seis parcelas medindo $10 \mathrm{~m} \times 200 \mathrm{~m}$, dentro das quais foram implantadas subparcelas de $10 \mathrm{~m} \times 10 \mathrm{~m}$ num total de 0,80 ha. Foram contabilizados 262 indivíduos distribuídos em 38 espécies, 23 gêneros e 27 famílias, 14 indivíduos foram identificados apenas em nível de família. As famílias com maior número de indivíduos foram Araceae (56), Bignoniaceae (45), Verbenaceae (35), Rubiaceae (21) e Sapindaceae (16). As famílias com maior número de espécies foram Rubiaceae (4), Bignoniaceae (3) e Cucurbitaceae (3). As espécies mais abundantes foram PhilodendronmelanorrhizumReitz (56), Petreasp. 1 (19), Petrea sp.2 (16), Paullinia sp. (16) e BauhiniamacrostachyaBenth. (15). A densidade total encontrada foi de 327,5 indivíduos por hectare e a frequência absoluta igual a 96,98 indivíduos por hectare. 0 índice de Shannon foi de 2,96, valor e o índice de equabilidade de Pielou foi igual a 0,75, valores que indicam alta diversidade na área amostrada e boa uniformidade na distribuição de espécies comparadas com outros estudos na Amazônia Brasileira.

PALAVRAS-CHAVE:Florística. Área de preservação permanente. Amazônia.

\section{ABSTRACT}

This study aimed to evaluate the floristic and ecological aspects of lianas occurring in the forest fragment of the Raimundolrineu Serra Environmental Protection Area, in Rio Branco, Acre. The floristic inventory was carried out with allocation of six plots measuring $10 \mathrm{~m} \times 200 \mathrm{~m}$, where plots of $10 \mathrm{~m} \times 10 \mathrm{~m}$ were implanted in a total of 0.80 ha. There were 262 individuals distributed in 38 species, 23 genders and 27 families, 14 individuals were identified only at family level. The families with the highest number of individuals were Araceae (56), Bignoniaceae (45), Verbenaceae (35), Rubiaceae (21) and Sapindaceae (16). The families with the highest number of species were Rubiaceae (4), Bignoniaceae (3) and Cucurbitaceae (3). The most abundant species were the Philodendronmelanorrhizum Reitz (56), Petrea sp. 1 (19), Petrea sp. 2 (16), Paullinia sp. (16) and Bauhinia macrostachyaBenth. (15). The total density found was 327.5 individuals per hectare and the absolute frequency equal to 96.98 individuals per hectare. The Shannon index was 2.96, and the Pielou equability index was 0.75 , which indicates high diversity in the sampled area and good uniformity in the species distribution when compared to other studies in the Brazilian Amazon.

KEY WORDS: Floristic. Permanent preservation area. Amazon.

\section{RESUMEN}

Este estudio tuvo como objetivo evaluar los aspectos florísticos y ecológicos de las lianas que ocurren en el fragmento forestal del Área de Protección Ambiental Raimundo Irineu Serra, en Rio Branco, Acre. Se produjo un inventario florístico con la asignación de seis parcelas con medidas de $10 \mathrm{~m} \times 200 \mathrm{~m}$, dentro de las cuales se implantaron subparcelas de $10 \mathrm{~m} \times 10 \mathrm{~m}$, que totalizaban 0,80 ha. Se contabilizó 262 individuos distribuidos en 38 especies, 23 géneros y 27 familias. 14 individuos fueron identificados sólo hasta en nivel de la familia. Las familias con el mayor número de individuos fueron Araceae (56), Bignoniaceae (45), Verbenaceae (35), Rubiaceae (21) y Sapindaceae (16). Las familias con el mayor número de especies fueron Rubiaceae (4), Bignoniaceae (3) y Cucurbitaceae (3). Las especies más abundantes fueron PhilodendronmelanorrhizumReitz (56), Petreasp. 1 (19), Petreasp. 2 (16), Paulliniasp. (16) y BauhiniamacrostachyaBenth. (15). La densidad total encontrada fue 327,5 de individuos por hectárea y la frecuencia absoluta fue igual a 96,98 individuos por hectárea. El índice de Shannon fue de 2,96, valor y el índice de equidad de Pielou fue igual a 0,75 , valores que indican una alta diversidad en el área estudiada y una buena uniformidad en la distribución de especies en comparación con otros estudios en la Amazonia Brasileña.

PALABRAS CLAVE: Florística. Área de conservación permanente. Amazônia. 


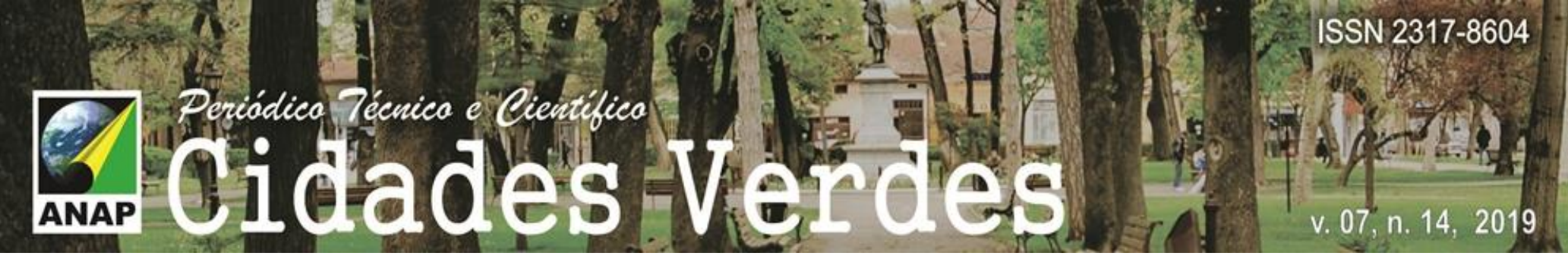

\section{INTRODUÇÃO}

A rápida expansão das cidades tem ocasionado a remoção da vegetação nativa para dar lugar às construções, muitas vezes levando ao surgimento de fragmentos florestais rodeados por uma matriz urbana (ARAÚJO, 2016; MELO et al., 2011). Um efeito comumente observado em decorrência da fragmentação florestal é o aumento do número de espécies lianescentes (ZHUO e CAO, 2010).

Entende-se como lianas ou trepadeiras os vegetaislenhosos ou herbáceos cujas sementes se desenvolvem no solo, mas a parte aérea depende de outras plantas para fornecer suporte físico e assim crescer em altura em direção à copa da planta suporte em busca de luz (QUARESMA e JARDIM, 2014; BRANDES e BARROS, 2008).

Segundo Pireset al.(2006), as espécies trepadeiras são plantas características de áreas perturbadas em decorrência da maior penetração de luz solar beneficiar seu desenvolvimento. Em comunidades florestais as lianas atuam de forma significativa nos processos de regeneração e sucessão em bordas e clareiras (SCHNITZER e BONGERS 2002, LAURENCE et al. 2001). Chazdon (2012) postula que com o fechamento do dossel, a tendência é que árvores, arbustos e lianas intolerantes à sombra sejam suprimidas, enquanto que as espécies umbrófilas são recrutadas no sub-bosque e no dossel e se estabelecem como mudas. A regeneração vigorosa de lianas em condições de boa luminosidade encontradas em florestas degradadas, secundárias e em clareiras pode resultar em uma competição não apenas por luz, mas também por nutrientes abaixo do solo, limitando o crescimento de plântulas, podendo atuar de forma seletiva na dinâmica da comunidade florestal ao interferir nas condições de vida dos demais componentes (SCHNITZER et al., 2012; PHILLIPS et al., 2005).

Apesar de sua importância para a dinâmica florestal, no Brasil os inventários florísticos raras vezes incluem lianas, havendo escassez de trabalhos nessa área e apontando a necessidade de mais estudos florísticos e ecológicos tenho como foco esse grupo de plantas (QUARESMA e JARDIM, 2016). O conhecimento da diversidade e estrutura das lianas de uma floresta torna-se uma importante ferramenta para o planejamento de ações de manejo, servindo para complementar estudos a cerca do efeito de perturbações e determinação de estágio de sucessão florestal (REZENDE et al., 2007).

A situação da Área de Proteção Ambiental Raimundo Irineu Serra (APARIS), por ser considerada uma unidade de conservação sob forte pressão antrópica devido a sua localização no perímetro urbano do município de Rio Branco, torna necessária a realização de estudos para o conhecimento de sua flora, que possam servir de contribuição para posteriores atividades de manejo e conservação.

\section{OBJETIVOS}

Diante do exposto, o objetivo deste estudo foi analisar a composição florística e a diversidade das lianas no maior fragmento florestal contínuo existente na APARIS, visando um maior conhecimento sobre os aspectos relacionados com a ecologia deste grupo de plantas.

\section{METODOLOGIA}

A Área de Proteção Ambiental Raimundo Irineu Serra possui uma extensão de aproximadamente 908,7 ha (Figura 1), das quais cerca de $30 \%$ são cobertas por fragmentos florestais em diversos estádios de regeneração (CARVALHO et al., 2010).Os remanescentes florestais são classificados, quanto a sua tipologia, 


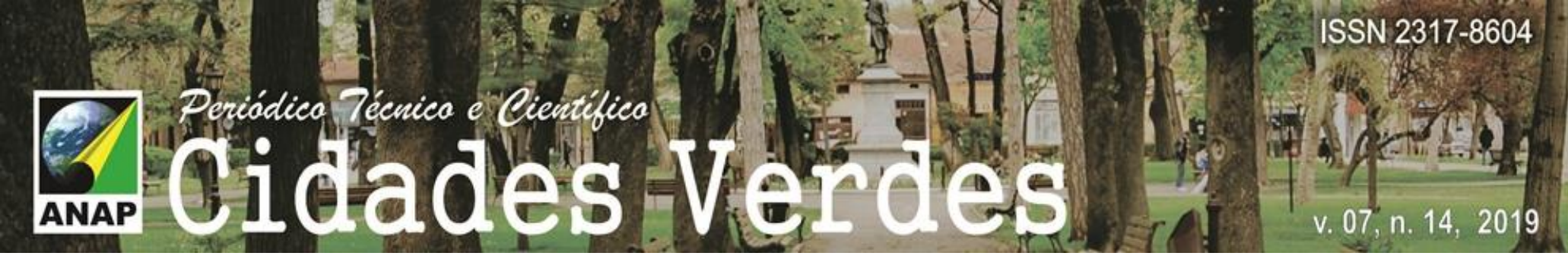

como Floresta Ombrófila Aberta com Palmeiras, os solos são do tipo luvissolo hipocrômico órtico, sendo pouco profundos e com tendência à erosão,o relevo tem variações altimétricas de $140 \mathrm{~m}$ a $270 \mathrm{~m}$ (ACRE, 2006). O clima no local, segundo a classificação de Koppen-Geiger, e do tipo Af, equatorial ou tropical úmido. A média pluviométrica anual varia entre $1877 \mathrm{~mm}$ e $1982 \mathrm{~mm}$ e a temperatura média anual varia de $24,7^{\circ} \mathrm{C}$ a $25,1^{\circ} \mathrm{C}$ (ACRE, 2006). O Levantamento de dados foi realizado em agosto de 2010.

Figura 1 - Localização da Área de Proteção Ambiental Raimundo Irineu Serra.

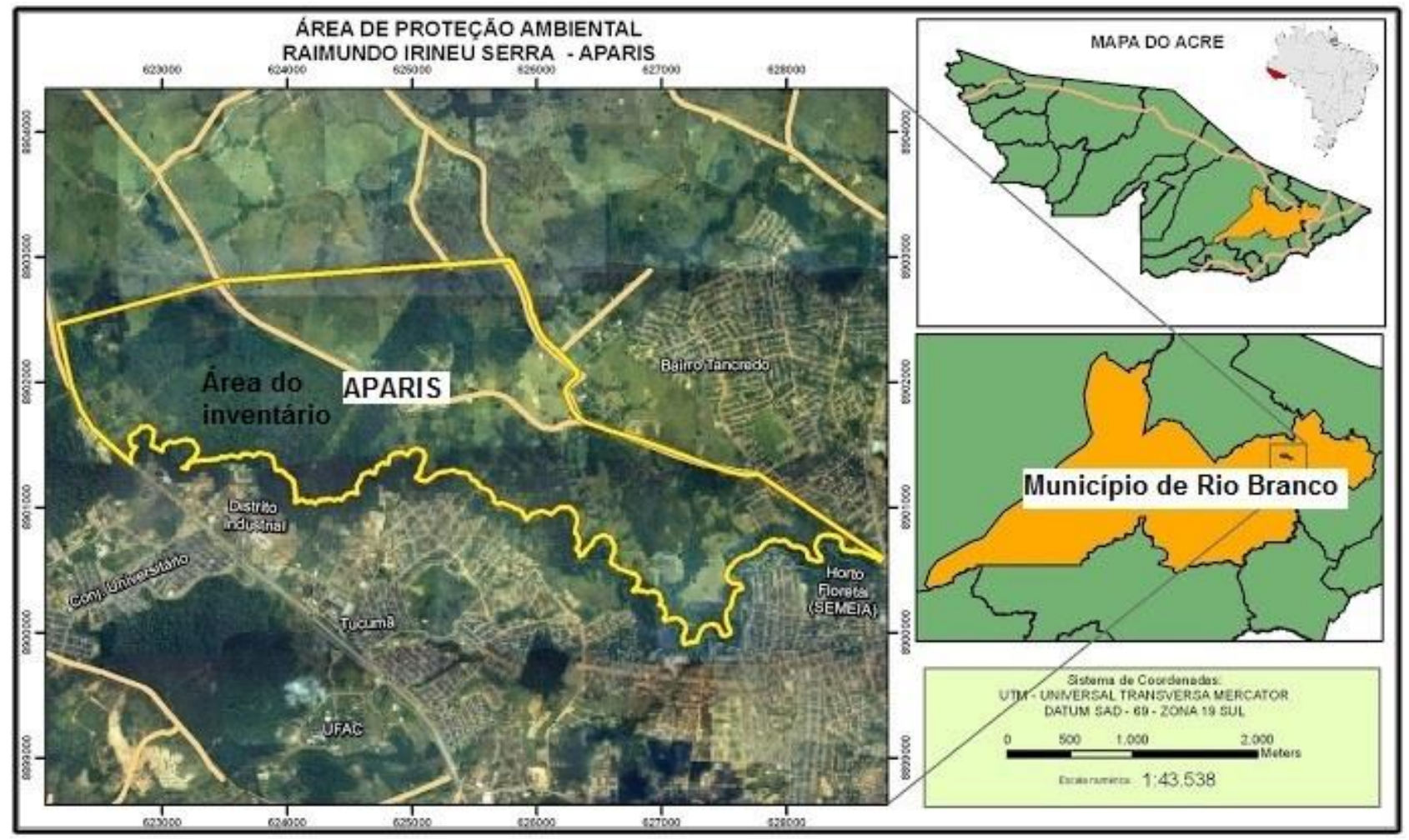

Fonte: Secretaria de Meio Ambiente do Acre - SEMA.

O levantamento florístico foi realizadono maior fragmento florestal contínuo existente na APA, com área estimada em pouco mais de 200 ha.Foram alocadas quatro parcelas retangulares medindo $200 \mathrm{~m} \times 10 \mathrm{~m}$ $\left(2000 \mathrm{~m}^{2}\right)$, distando $500 \mathrm{~m}$ uma da outra. Dentro de cada parcela foram alocadas 20 subparcelas contínuas de $10 \mathrm{~m} \times 10 \mathrm{~m}$. Ao todo área abrangida por este inventário foi de 0,80 ha.

Os indivíduos inventariados foram identificados em campo por um especialista parabotânico e posteriormente, com auxílio de literatura científica eda lista do banco de dados do Missouri Botanical Garden do The New York Botanical Garden realizou-se a confirmação das identificações e da nomenclatura.Para análise de heterogeneidade florística foram utilizados o índice de diversidade de Shannon-Wiener (MAGURRAN 1988) e de equitabilidade (PIELOU 1975):

a) Índice de Diversidade de Shannon-Wiener $\left(\mathrm{H}^{\prime}\right)$

O índice de diversidade de Shannon-Wiener é obtido pela somatória do produto entre a abundância relativa de cada espécie e o logaritmo neperiano desse mesmo valor.

$H^{\prime}=\frac{\left[N \cdot \ln (H)-\sum_{i=1}^{s} n_{i} \cdot \ln \left(n_{i}\right)\right]}{N}$ 
Em que:

$N$ = número total de indivíduos amostrados;

$n_{i}=$ número de indivíduos amostrados da i-ésima espécie;

$S=$ número de espécies amostradas;

In = logaritmo de base neperiana (e).

b) Índice de Equabilidade de Pielou

Este índice possui um intervalo de [0 a 1], onde 1 representa a máxima diversidade, ou seja, todas espécies são igualmente abundantes.

$\mathrm{J}=\frac{\mathrm{H}^{\prime}}{\mathrm{Hmax}}$

Em que:

$H \max =\ln (\mathrm{S})$;

$\mathrm{H}^{\prime}$ = índice de diversidade de Shannon-Wiener.

Para o estudo fitossociológico calculou-se as densidades absoluta e relativa de acordo com as fórmulas:

a) Densidade absoluta:

$$
\mathrm{DA}=\frac{\mathrm{n}}{\mathrm{ha}}
$$

Em que:

$D A=$ densidade absoluta da i-ésima espécie, em número de indivíduos por hectare;

$\mathrm{n}=$ número total de indivíduos amostrados de cada espécie.

ha= hectare

b) Densidade relativa:

$\mathrm{DR}=\frac{\mathrm{n} / \mathrm{ha}}{\mathrm{N} / \mathrm{ha}} \cdot 100$

Em que:

$\mathrm{DR}=$ densidade relativa (\%).

$n=$ número total de indivíduos amostrados de cada espécie;

$N=$ número total de indivíduos mostrados, de todas as espécies do levantamento

\section{RESULTADOS}

Foram amostrados 262 indivíduos, destes, catorze foram identificados apenas em nível de família Tabela 1. As espécies identificadas somaram 38, distribuídas em 21 gêneros e 27 famílias (Figura 2).

Tabela 1- Lista das espécies lianas encontradas durante o levantamento florístico de um fragmento da Área de Proteção Ambiental Raimundo Irineu Serra, Rio Branco, Acre. 


\begin{tabular}{|c|c|c|c|c|}
\hline Nome científico & & $\begin{array}{l}\text { Número } \\
\text { indivíduos }\end{array}$ & de DA & DR \\
\hline Philodendron melanorrhizum Reitz & Araceae & 56 & 70,00 & 21,37 \\
\hline ni 1 & Bignoniaceae & 36 & 45,00 & 13,74 \\
\hline Petrea blanchetiana Schauer. & Verbenaceae & 19 & 23,75 & 7,25 \\
\hline Petrea sp. 1 & Verbenaceae & 16 & 20,00 & 6,11 \\
\hline Paullinia elegans Camb. & Sapindaceae & 15 & 18,75 & 5,73 \\
\hline Bauhinia macrostachya Benth. & Caesalpiniaceae & 14 & 17,50 & 5,34 \\
\hline ni 2 & Apocynaceae & 9 & 11,25 & 3,44 \\
\hline Psychotria sp. & Rubiaceae & 9 & 11,25 & 3,44 \\
\hline Mussatia sp. & Bignoniaceae & 8 & 10,00 & 3,05 \\
\hline Uncaria sp. & Rubiaceae & 8 & 10,00 & 3,05 \\
\hline ni 3 & Solanaceae & 6 & 7,50 & 2,29 \\
\hline Omphalea diandra $\mathrm{L}$. & Euphorbiaceae & 5 & 6,25 & 1,91 \\
\hline Humirianthera ampla (Miers.) & Icacinaceae & 5 & 6,25 & 1,91 \\
\hline Davillasp. & Dilleniaceae & 4 & 5,00 & 1,53 \\
\hline Doliocarpus dentatus(Aubl.) Standl. & Dilleniaceae & 4 & 5,00 & 1,53 \\
\hline ni 04 & Hippocrateaceae & 4 & 5,00 & 1,53 \\
\hline ni 05 & Malpighiaceae & 4 & 5,00 & 1,53 \\
\hline Celtis schippii Standl. & Ulmaceae & 4 & 5,00 & 1,53 \\
\hline Cissus rhombifoliaVahl. & Vitaceae & 4 & 5,00 & 1,53 \\
\hline ni 06 & Polygalaceae & 3 & 3,75 & 1,15 \\
\hline ni 07 & Polygonaceae & 3 & 3,75 & 1,15 \\
\hline Clematis dioica var. brasiliana & Ranunculaceae & 3 & 3,75 & 1,15 \\
\hline Smilax japecanga Griseb & Smilacaceae & 3 & 3,75 & 1,15 \\
\hline ni 08 & Curcubitaceae & 2 & 2,50 & 0,76 \\
\hline ni 09 & Quiinaceae & 2 & 2,50 & 0,76 \\
\hline ni 10 & Rubiaceae & 2 & 2,50 & 0,76 \\
\hline Uncaria guianensisJ. F. Gmel. & Rubiaceae & 2 & 2,50 & 0,76 \\
\hline Triumfettasp. & Tiliaceae & 2 & 2,50 & 0,76 \\
\hline Tanaecium nocturnum (Barb.Rodr.) Bureau\&K.Schum. & Bignoniaceae & 1 & 1,25 & 0,38 \\
\hline Anaxagorea dolichocarpa Sprague \& Sandwith & Annonaceae & 1 & 1,25 & 0,38 \\
\hline ni 11 & Aristolochiaceae & 1 & 1,25 & 0,38 \\
\hline Guraniasp. & Curcubitaceae & 1 & 1,25 & 0,38 \\
\hline ni 12 & Curcubitaceae & 1 & 1,25 & 0,38 \\
\hline Dalbergia frutescens (Vell.) Britton. & Fabaceae & 1 & 1,25 & 0,38 \\
\hline Chondodendron platyphyllum (A. St.-Hil.) Miers. & Menispermaceae & 1 & 1,25 & 0,38 \\
\hline Passiflora sp. & Passifloraceae & 1 & 1,25 & 0,38 \\
\hline ni 13 & Rhamnaceae & 1 & 1,25 & 0,38 \\
\hline \multirow[t]{2}{*}{ ni 14} & Sapindaceae & 1 & 1,25 & 0,38 \\
\hline & & 262 & 100 & 327,5 \\
\hline
\end{tabular}

ni = espécie não identificada. 


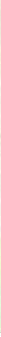

As famílias que apresentaram maior número de indivíduos foram Araceae (56), Bignoniaceae (45), Verbenaceae (35), Rubiaceae (21), Sapindaceae (16) e Fabaceae (15) as demais famílias apresentaram entre um e oito indivíduos (Figura 2).

Figura 2 - Número de indivíduos por família encontrados no fragmento florestal da Área de Proteção Ambiental Raimundo Irineu Serra.

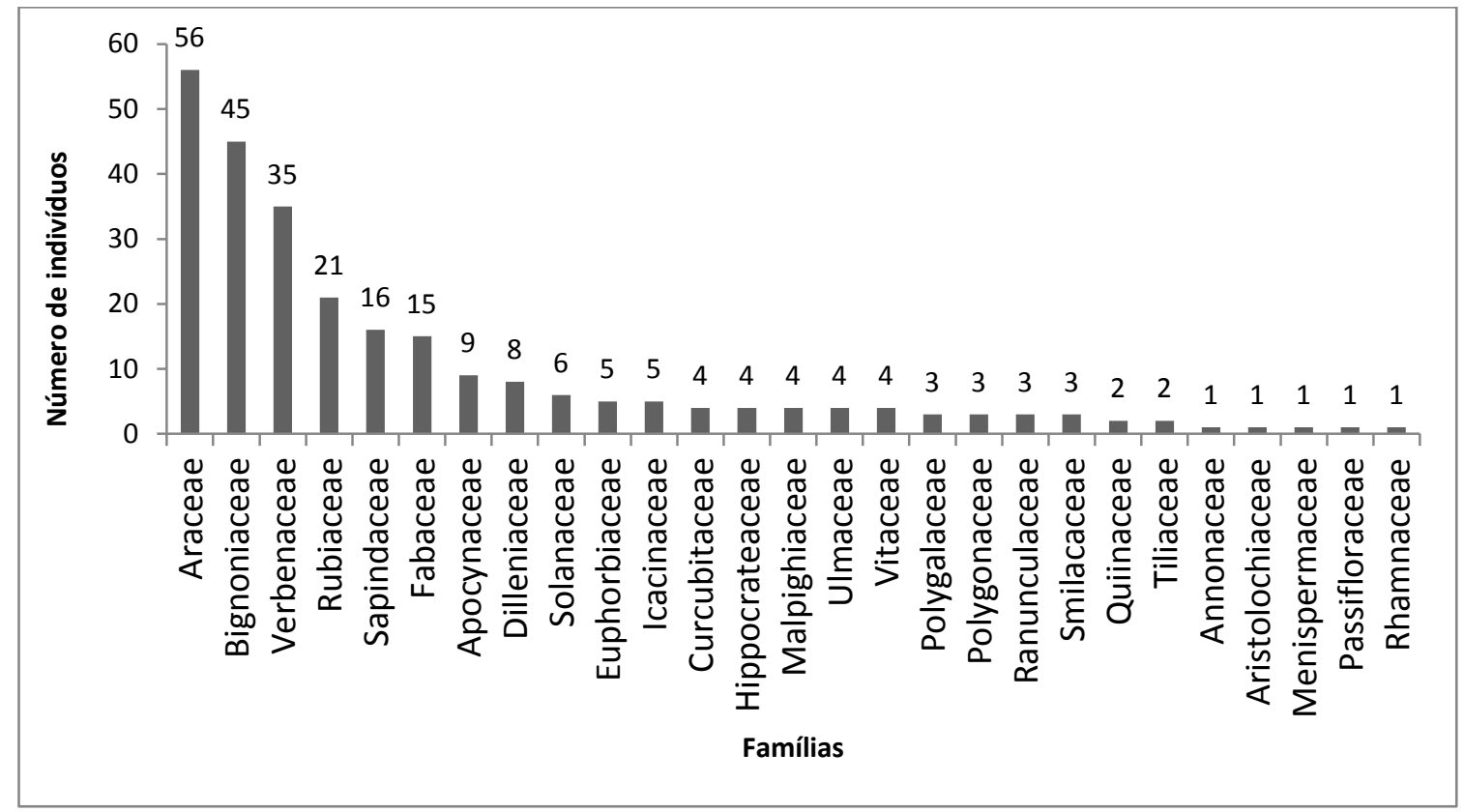

As famílias com maior número de espécies foram Rubiaceae (quatro), Bignoniaceae (três) e Cucurbitaceae (três) conforme ilustra a figura a 3. 


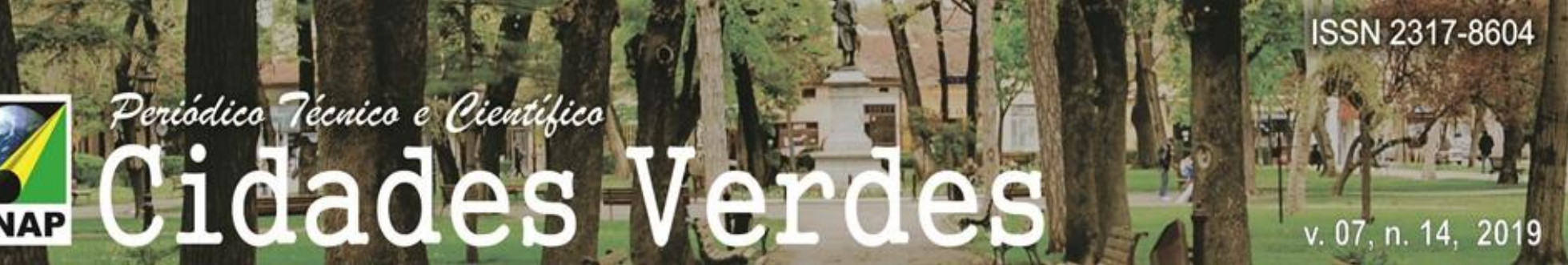

Figura 3 - Número de espécies por família encontradas no fragmento florestal da Área de Proteção Ambiental Raimundo Irineu Serra.

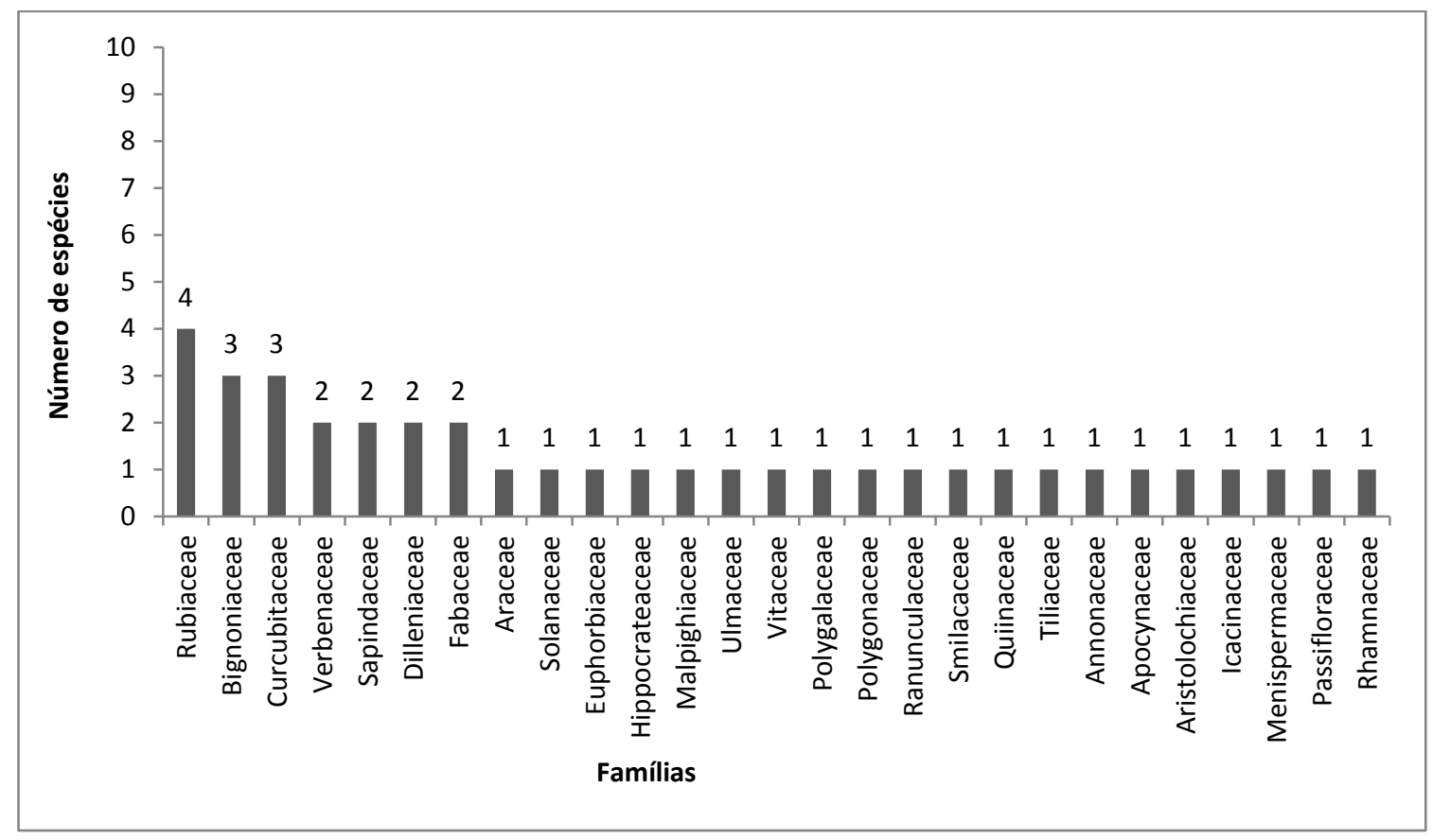

A densidade total encontrada foi de 327,5 indivíduos por hectare e a frequência absoluta igual a 96,98 indivíduos por hectare (Tabela 2). 0 índice de Shannon encontrado foi de 2,96 e o índice de equabilidade de Pielou igual a 0,75.A espécie Philodendron melanorrhizum Reitz apresentou a maior número de indivíduos, tendo sido responsável por 21,29\% do total da amostra com densidade relativa de 21,37.

Comparando os resultados com levantamentos similares observou-se que tanto o número de família, gênero e espécie encontrados nesse estudo foram superiores aos encontrados por Quaresma e Jadim (2016) e Oliveira et al. (2008) em áreas também situadas na região amazônica (Tabela 1).Em áreas mais extensas de florestas do sudeste esses valores foram superiores. Fatores como a tipologia florestal, tipos de solo e o histórico de perturbação podem estar relacionados com essa diferença.

Para Davis e Richards (1933), a intensidade luminosa não seria o principal fator a agir sobre a redução da ocorrência de lianas, e sim a umidade do solo, segundo eles indivíduos lianescentes tendem a preferir solos mais argilosos que retêm melhor a umidade. No presente estudo, a área amostral está situada sobre uma mancha de luvissolo, típico de terraços fluviais (ACRE, 2006; PEREIRA et al. 2010), a textura argilosa com baixa permeabilidade pode ter proporcionado condições favoráveis para a colonização por parte das lianas. 


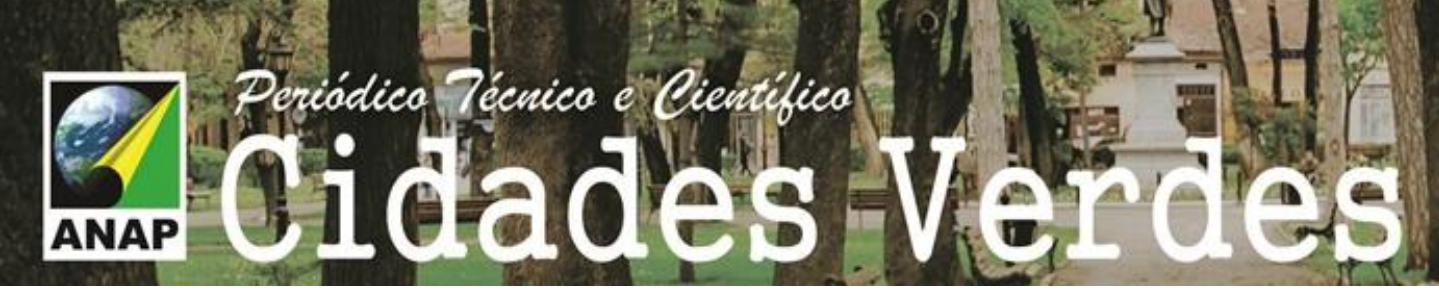

Tabela 2 - Número de espécies, gêneros e famílias de lianas em diferentes áreas florestais na região amazônica.

\begin{tabular}{lcccccc}
\hline Localidade & Tipologia florestal & $\begin{array}{c}\text { Área } \\
\text { (ha) }\end{array}$ & $\begin{array}{c}\text { Número de } \\
\text { famílias }\end{array}$ & $\begin{array}{c}\text { Número de } \\
\text { gêneros }\end{array}$ & $\begin{array}{c}\text { Número de } \\
\text { espécies }\end{array}$ & Referências \\
\hline $\begin{array}{l}\text { Rio } \\
\text { Branco/AC }\end{array}$ & $\begin{array}{r}\text { Floresta ombrófila aberta com } \\
\text { palmeiras }\end{array}$ & 0,80 & 27 & 23 & 38 & Este estudo \\
Maracanã/PA & "Floresta Seca" de restinga & 0,50 & 10 & 15 & 17 & $\begin{array}{c}\text { Quaresma e Jardim. } \\
\text { (2016) }\end{array}$ \\
Manaus/AM & Floresta ombrófila densa & 3 & 9 & 10 & 13 & Oliveira et al. (2008) \\
\hline
\end{tabular}

Observou-se que em 32,14\% das famílias registrou-se apenas um indivíduo, o que fica abaixo do referido por Oliveira et al. (2008), segundo os autores nos levantamentos fitossociológicos de lianas na Amazônia a porcentagem de famílias com apenas um indivíduo é maior, variando de $40 \%$ a $60 \%$.

Nenhuma família apresentou mais de quatro espécies. A família Rubiaceae foi a que reuniu maior número de espécies (4), seguida por Bignoniaceae (3) e Cucurbitaceae (3). A família Rubiaceae é formada por mais de 125 gêneros e 1.400 espécies, é a quarta família com maior número de espécies, sendo comum que esta figure em levantamentos florísticos por sua ampla distribuição geográfica (DELPRETE e JARDIM, 2012).

A família Bignoniaceae figura entre as famílias de maior riqueza específica em diversos estudos com lianas entre eles Tibiriçá et al. (2006), Quaresma e Jardim (2015), Carneiro e Vieira (2012) e Vargas et al. (2013). Segundo Gentry (1991) esta família é uma das mais abundantes em espécies nas regiões neotropicais, no entanto, no Brasil a taxonomia das bignoniaceaes não arbóreas ainda é pouco estudada (SCUDELLER e CARVALHO-OKANO, 1998).

A família Cucurbitaceae assim como Bignoniaceae, esteve entre as mais ricas em espécies no levantamento realizado por Tibiriçá et al. (2006).Segundo Schaefer e Renner (2011) esta família possui distribuição tropical e subtropical, é composta por 97 gêneros e entre 940 e 980 espécies. No Brasil registrou-se a ocorrência de 30 gêneros e 147 espécies de cucurbitaceaes em diferentes fisionomias (KLEIN e LIMA, 2010).

A família Araceae na qual o número total de indivíduos amostrados corresponde a $21,37 \%$ do total da amostra foi registrada apenas uma espécie, Philodendron melanorrhizum Reitz conhecido como cipó-preto, cipó-imbé ou cipó-rama. Esta espécie possui dispersão zoocórica, suas sementes são consumidas principalmente por primatas e marsupiais e possuem alta porcentagem de germinação após serem expelidas nas fezes desses animais (VIEIRA e ZAR, 1999). Algumas espécies de formigas também realizam a dispersão dessas sementes ao separarem-nas das fezes dos primatas, realizando uma dispersão secundária (PIZO e OLIVEIRA, 2000). As formigas podem alterar o padrão de deposição das sementes e o sucesso reprodutivo da espécie agindo sobre a distribuição espacial (ROBERT e HEITHAUS, 1986).Essas possibilidades de dispersão aliada às condições ambientais favoráveis podem ter facilitado a distribuição do P. melanorhizumna área.

Verbenaceae destacou-se por ser a terceira maior família em número de indivíduos (35) mesmo com apenas duas espécies. Segundo Salimena et al. (2014) o Brasil é o país onde se observou a maior riqueza desta família, 16 gêneros e 290 espécies, sendo 191 endêmicas. Ao todo a família possui cerca de 32 gêneros e 480 espécies, com distribuição neotropical (ATKINS et al., 2004). Neste estudo o único gênero registrado para a família foi o gênero Petrea. De acordo com Rueda (1994) o gênero Petrea possui cerca de 14 espécies conhecidas das quais três são fósseis, originárias da América Tropical. 


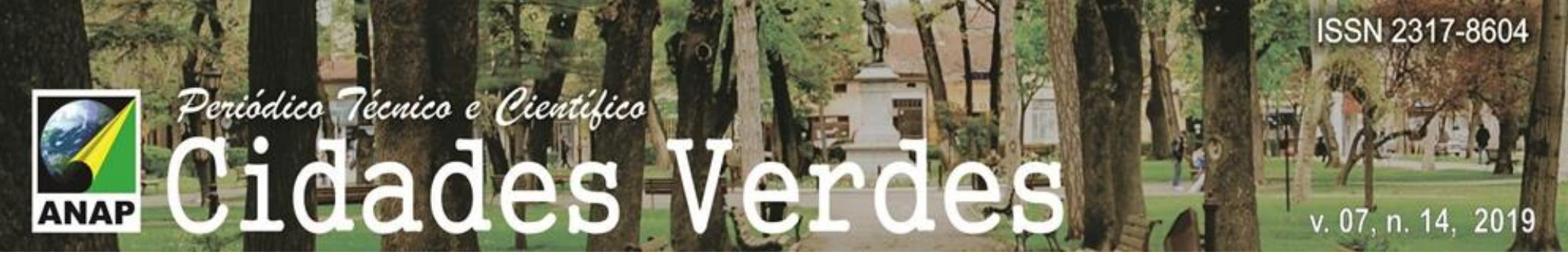

$O$ índice de diversidade encontrado $(2,96)$ foi próximo ao registrado por Oliveira e Amaral (2008) em floresta de platô da Amazônia Central encontraram H'=2,5. No estudo realizado por Oliveira et al. (2011) os autores registraram índices de diversidade variando de 2,47 a 0 em diferentes tipologias florestais na transição Amazônia-Cerrado no Mato Grosso.O índice de equabilidade de Pielou igual a 0,75 indicando uma boa uniformidade na distribuição das espécies. Oliveira e Amaral (2008) encontraram uma equabilidade máxima igual a 0,95 analisando uma comunidade de lianas em floresta de platô, 0,92 em floresta de vertente e 1 em floresta de baixio da Amazônia Central. Segundo os mesmo autores, os diferentes métodos de amostragem, área de estudo e critério de inclusão dificultam a comparação de resultados envolvendo índices de diversidade no estudo de lianas.

\section{CONCLUSÃO}

Foramregistradas 38 espécies de lianas distribuídas em 23 gêneros e 27 famílias botânicas. Algumas famílias foram representadas por apenas uma espécie.

Apesar da pressão exercida com crescimento da cidade de Rio Branco, a comunidade de lianas da APA Raimundo Irineu Serra apresentou índices de diversidade e equitabilidade bem próximos e até mesmo superiores aos encontrados em outros estudos, inclusive em áreas sem um histórico de intervenção tão recente. A heterogeneidade de espécies observada em uma pequena porção do fragmento analisado demonstra a importância das lianas na composição florestal local.

\section{REFERÊNCIAS}

ACRE. Governo do Estado do Acre. Programa Estadual de Zoneamento Ecológico-Econômico do Estado do Acre. Zoneamento Ecológico-Econômico do Acre Fase II: documento Síntese - Escala 1:250.000. Rio Branco: SEMA. 354 p. 2006.

ARAÚJO, E. Identificação de Ilhas de Calor na Cidade de Campina Grande-PB utilizando Sensoriamento Remoto. Revista Brasileira de Geografia Física,Recife, v. 9, n. 2, p.? 2016.

ATKINS, S. Verbenaceae. In: Kadereit, J. W. (ed.). The families and genera of flowering plants.Vol. 7. Springer-Verlag: Berlin, 2004, pp. 449-468.

BRANDES, A.F.N.; BARROS, C.F. Anatomia do lenho de oito espécies de lianas da família Leguminosae ocorrentes na Floresta Atlântica. Acta Botanica Brasilica, Belo Horizonte, v.22, n.2, p.465-480, 2008.

CARVALHO, A.; FERREIRA, E.JL.; TUCKER, J.D. Comparações florísticas e estruturais entre comunidades de palmeiras em fragmentos de floresta primária e secundária da Área de Proteção Ambiental Raimundo Irineu Serra- Rio Branco, Acre, Brasil. Acta amazônica,Manaus, v. 40, n. 4, p. 657-666, 2010.

CHAZDON, R. Regeneração de florestas tropicais. Boletim do Museu Paranaense Emílio Goeldi, Belém, v. 7, n. 3, p.195-218, 2012.

DAVIS, T.A.W.; RICHARDS, P.W. The vegetation of Moroballi Creek, British Guiana: an ecological study of a limited area of tropical rain forest.Part I. Journal of Ecology, London, v. 21p. 350-384, 1933.

DELPRETE, P.G.; JARDIM, J.G. Systematics, taxonomy and floristics of Brazilian Rubiaceae: an overview about the current status and challenges. Rodriguésia, Rio de Janeiro, v. 63, n. 1, p. 101-128. 2012. 
GENTRY, A.H.The distribution and evolution of climbing plants.In:PUTZ, F.E. MOONEY, H.A. The biology of vines. Cambridge: University Press, p. 3-49, 1991.

KLEIN, V. L. G.; LIMA, L. F. P. Cucurbitaceae.In: Lista de Espécies da Flora do Brasil. Jardim Botânico do Rio de Janeiro. 2011. Disponível em: <http://floradobrasil.jbrj.gov.br/>. Acessoem: 03 fev. de 2017.

LAURENCE, W.F.; PERÉZ-SAlICRUP, D.; DALEMÔNICA, P.; FEARNSIDE, P.M.; D’ANGELO, S.; JEROZOLINSKI, A.; POHL, L. \& LEVEJOY, T.E. Rain forest fragmentation and the structure of Amazonian liana communities. Journal of Ecology, London, v. 82, p. 105-116, 2001.

MAGURRAN , A. E. Diversidad Ecologica y su Medición. Espanha: Ediciones Vedra. 1988.

MELO, A. G. C.; CARVALHO, D. A. CASTRO, G. C.; MACHADO, E. L. M. Fragmentos florestais urbanos. Revista Cientifica Eletrônica de Engenharia Florestal, Garça, v. 17, n. 1, 2011.

OLIVEIRA, A. N.; AMARAL, I. L.; RAMOS, M. B. P.; FORMIGA, K. M. Aspectos florísticos e ecológicos de grandes lianas em três ambientes florestais de terra firme na Amazônia Central. Acta Amazonica,Manaus, v.38, n.3, p. 421 - 430, 2008.

PEREIRA, J. M., E. A. ARAúJO \& N. G. BARDALES. 2010. Características gerais do Acre. In: Reunião Brasileira de Classificação e Correlação de Solos (RCC): solos sedimentares em sistemas amazônicos - potencialidades e demandas de pesquisa, 9. 2010. Anais... v. 9, n. 1, p. 11-20, 2010.

PIELOU, E. C. Ecological diversity. New York: Wiley, 1975. 165 p.

PIRES, A. S.; FERNANDEZ, F. A. S.; BARROS, C. S. Vivendo em um mundo em pedaços: efeitos da fragmentação florestal sobre comunidades e populações animais. In: Rocha, C. F. D.; Bergallo, H. G.; Alves, M. A. S.; Sluys, M. V. (Eds.): Biologiada conservação: essências.São Carlos: Rima, p. 231-260. 2006.

PHILLIPS, O.L.; VÁSQUEZ MARTínEZ, R.; MONTEAGUdO MENDOZA, A.; BAKER, T.R. \& NÚNÊZ VARGAS, P. Large lianas as hyperdynamic elements of the tropical forest canopy. Journal of Ecology, London, v. 86, p. 1250-1258, 2005.

PIZO, M. A.; OLIVEIRA, P.S.The Use of Fruits and Seeds by Ants in the Atlantic Forest of Southeast Brazil. Biotropica, Belo Horizonte, v. 32, n. 4, p: $851-861,2000$.

QUARESMA, A.C.; JARDIM, M.A.G. Floristic composition and spatial distribution of vascular epiphytes in the restingas of Maracanã, Brazil.Acta Botanica Brasilica, Belo Horizonte, v.28, n.1, p.68-75, 2014.

QUARESMA, A. C.; JARDIM, M. A. G. Formações florestais de restinga e relações ecológicas com lianas. Revista Árvore,Viçosa, v. 39, n. 2, p. 255-261, 2016.

REZENDE, A.A.; RANGA, N.T.; PEREIRA, R.S. Lianas de uma floresta estacional semidecidual, Município de Paulo de Faria, norte do estado de São Paulo, Brasil. Revista Brasileira de Botânica,São Paulo, v. 30, n. 3, p: 451-461, 2007.

ROBERTS, J.T.; HEITHAUS, E.R. Ants rearrange the vertebrate generated seed shadow of a neotropical fig tree. Journal of Ecology, London, v. 67, p. 1046-1051, 1986.

RUEDA, R.Systematics and Evolution of the Genus Petrea (Verbenaceae).Annals of the Missouri Botanical Garden, v. 81, n. 4, p. 610-652, 1994.

SALIMENA, F.R.G.; MÚlgURA, M. 2014. Lippia. In:Lista de espécies da flora do Brasil. Jardim Botânico do Rio de Janeiro. Disponível em <http://floradobrasil. jbrj.gov.br/jabot/floradobrasil/FB15170> Acesso em 08 de março de 2017. 


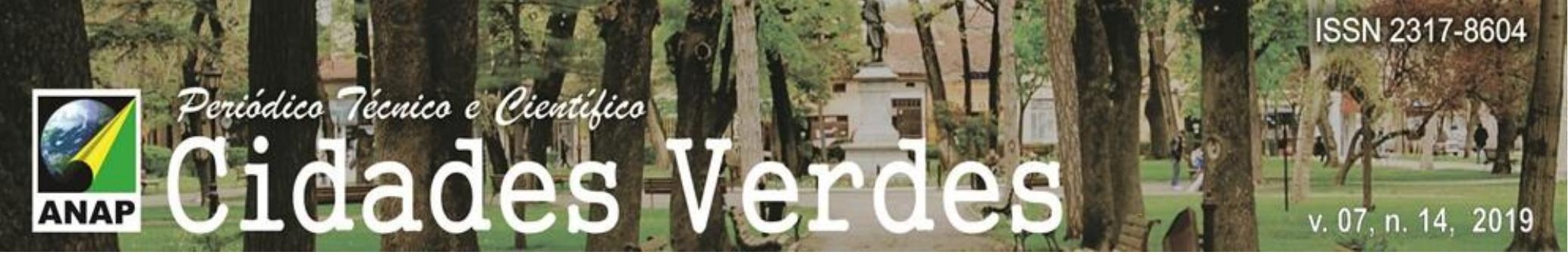

SCHNITZER, S. A.; BONGERS F. 2002.The ecology of lianas and their role in forests.Trends in Ecology and Evolution,London, v. 17, n. 5, p. 223-230, 2002.

SCHNITZER, S.A., MANGAN, S.A., DALLING, J.W., BALDECK, C.A., HUBBELL, S.P., LEDO, A., MULLER-LANDAU, H., TOBIN, M.F., AGUILAR, S., BRASSFIELD, D., HERNANDEZ, A., LAO, S., PEREZ, R., VALDES, O., YORKE, S.R. Liana abundance, diversity, and distribution on Barro Colorado Island, Panama. PloS One, v. 7, n. 12, 2012.

SCHAEFER, H.; RENNER, S. S. 2011.Phylogenetic relationships in the order Cucurbitales and a new classification of the gourd family (Cucurbitaceae).Taxon, Reino Unido, v. 60: 122-138.

SCUDELlER, V.V.; CARVALHO-OKANO, R.M. Bignonieae (Bignoniaceae) no Parque Estadual do Rio Doce, Minas Gerais, Brasil. Iheringia, Série Botânica,Porto Alegre, v. 51, n. 1, p. 79-133, 1998.

TIBIRIÇÁ, Y.J.A.; COELHO, L.F.M.; MOURA, L.C. Florística de lianas em um fragmento de floresta estacional semidecidual, Parque Estadual de Vassununga, Santa Rita do Passa Quatro, SP, Brasil. Acta Botanica Brasilica, Belo Horizonte, v. 20, p. 339-346, 2006.

VARGAS, B. C.; ARAÚJO, G.M.; SCHIAVINI, I.; ROSA, P.O.; HATTORI, E.K.O. Florística de trepadeiras em floresta semidecidualem em mata ciliar no vale do rio Araguari, MG. Bioscience Journal,Umuarama, v. 29, n. 1, p. 185-197, 2013.

VIEIRA, E. M.; IZAR, P. Interactions between aroids and arboreal mammals in the Brazilian Atlantic rainforest.Plant Ecology, Uppsala, v. 145, p. $75-82,1999$.

ZHUO, S.; CAO, K.Contrasting cost-benefit strategy between lianas and trees in a tropical seasonal rain forest in southwestern China. Oecologia, Berlin, v.163, n. 3, p. 591-599, 2010. 